\title{
Clinical applications of polygenic breast cancer risk: a critical review and perspectives of an emerging field
}

\author{
Tatiane Yanes ${ }^{1,2^{*}}$ (D), Mary-Anne Young ${ }^{4}$, Bettina Meiser ${ }^{1}$ and Paul A. James ${ }^{3}$
}

\begin{abstract}
Polygenic factors are estimated to account for an additional 18\% of the familial relative risk of breast cancer, with those at the highest level of polygenic risk distribution having a least a twofold increased risk of the disease. Polygenic testing promises to revolutionize health services by providing personalized risk assessments to women at high-risk of breast cancer and within population breast screening programs. However, implementation of polygenic testing needs to be considered in light of its current limitations, such as limited risk prediction for women of nonEuropean ancestry. This article aims to provide a comprehensive review of the evidence for polygenic breast cancer risk, including the discovery of variants associated with breast cancer at the genome-wide level of significance and the use of polygenic risk scores to estimate breast cancer risk. We also review the different applications of this technology including testing of women from high-risk breast cancer families with uninformative genetic testing results, as a moderator of monogenic risk, and for population screening programs. Finally, a potential framework for introducing testing for polygenic risk in familial cancer clinics and the potential challenges with implementing this technology in clinical practice are discussed.
\end{abstract}

Keywords: Breast cancer, Polygenic risk score, Risk prediction

\section{Introduction}

Breast cancer is a common disorder with a strong hereditary contribution. Women with a first-degree relative with breast cancer have a twofold increased risk of developing the disease $[1,2]$. Much of the hereditary component of breast cancer remains unexplained, with pathogenic variants in moderate- and high-risk genes, such as BRCA1 and BRCA2 (BRCA1/2), accounting for less than $25 \%$ of the familial risk for the disease [3]. Recent studies suggest breast cancer is a complex disease, with polygenic factors accounting for a further $18 \%$ of the familial risk [4]. Currently, there is considerable debate over the clinical utility of polygenic information to assess breast cancer risk. Supporters of this technology argue that polygenic testing has the potential to provide risk information for a significant number of women who

\footnotetext{
* Correspondence: t.yanes@uq.edu.au

${ }^{1}$ Psychosocial Research Group, Prince of Wales Clinical School, Faculty of Medicine, UNSW Sydney, Sydney, NSW, Australia

${ }^{2}$ The University of Queensland Diamantina Institute, Dermatology Research

Centre, University of Queensland, Brisbane, QLD 4102, Australia

Full list of author information is available at the end of the article
}

would otherwise receive uninformative genetic testing results and further stratify risk for those with pathogenic variants in high- and moderate-risk genes [5-7]. Additionally, polygenic risk promises to revolutionize population screening programs by providing personalized risk assessments and risk management strategies $[8,9]$. However, it has also been argued that there is not enough evidence to support its implementation in clinical practice and that the test does not provide sufficient risk stratification to warrant implementation over traditional risk assessment tools [10-12]. Furthermore, there is no consensus for the implementation of this technology, such as which variants to include in the polygenic risk calculations. Despite these concerns, testing for polygenic breast cancer risk is now being implemented in clinical practice with several commercial genetic testing laboratory now offering the test $[13,14]$.

This article aims to review the evidence for testing for polygenic breast cancer risk and the different applications of the test, including for high-risk women with uninformative genetic testing results, as a modifier of

(C) The Author(s). 2020 Open Access This article is distributed under the terms of the Creative Commons Attribution 4.0 International License (http://creativecommons.org/licenses/by/4.0/), which permits unrestricted use, distribution, and 
monogenetic risk genes, and to guide population screening programs. This article also explores the potential benefits and limitations of this technology, as well as the challenges associated with implementing polygenic testing in clinical practice.

\section{Genome-wide association studies}

There have been over 100 different breast cancer genome-wide association studies (GWAS), which have collectively led to the identification of over 182 variants associated with breast cancer risk at the genomewide level of significance (Additional file 1) [15]. Given the small effect size of these variants, there has been an ongoing need to increase the sample sizes of GWAS to detect true genetic associations [16]. Increasingly large GWAS have been made possible due the establishment of international research consortia, including the Breast Cancer Association Consortium $(\mathrm{BCAC})$ and The Collaborative Oncological GeneEnvironment Study (COGS), formed in 2005 and 2009 respectively $[17,18]$.

The COGS combined data from multiple international consortiums with the goal of improving understanding of the genetic susceptibility to breast, ovarian, and prostate cancer, and designed a custom array, the Illumina iSelect (iCOGS) [18]. The first major breast cancer outcome from the COGS program was a GWAS that genotyped 52,675 cases and 49,436 controls of European ancestry [19]. Twenty-three of the 27 previously reported breast cancer associated loci were replicated using iCOGS and an additional 41 new breast cancer loci were identified. Further analysis of the full dataset suggested that more than 1000 loci are involved in breast cancer susceptibility [19].

In a follow-up study, a meta-analysis of 11 previous GWAS together with the data from the 41 studies genotyped in iCOGS was conducted. Data for 62,533 breast cancer cases and 60,976 controls of European ancestry was analyzed, leading to the identification of an additional 15 breast cancer associated variants along with replication of 71 of the 79 previously reported variants [20]. Consistent with previous GWAS, nearly all of these variants were located in intronic or intergenic regions of the genome, suggesting they are regulatory [20].

In the largest breast cancer GWAS to date, Michailidou et al. [4] genotyped 61,282 cases and 45,494 controls of European ancestry using the specifically developed Illumina OncoArray and results were also included in a meta-analysis of a further 61,695 cases and 60,480 controls from iCOGS and 11 other studies. This comprehensive analysis led to the identification of the 61 new breast cancer variants, and 49 out of the 102 previously reported loci showed evidence of association with breast cancer risk in the OncoArray dataset. Together, the 182 known loci were estimated to account for $18 \%$ of the familial risk for breast cancer [4].

There have been increasing efforts to conduct GWAS in non-European populations, with most of the reported breast cancer variants discovered in European cohorts [21-25]. Michailidou et al. [4] conducted a meta-analysis of 14,068 cases and 13,104 controls of East Asian ancestry. Out of 94 loci discovered in European populations, 50 showed evidence of association in the East Asian cohort. Similarly, five loci previously reported in Asian women showed evidence in the European cohort. However, such findings have not been consistent across different ethnic groups. For example, approximately 100 variants previously validated in European or Asian women did not provide comparable risk stratification in women of African ancestry, with 30 to $40 \%$ of variants conferring risk in one population but appearing to be protective in another [26]. These findings highlight the need to conduct further studies to assess the association of breast cancer variants in non-European populations and to identify novel variants that confer breast cancer risk in those populations.

Most GWAS to date have been unselected for breast cancer subtype and as a result include a majority of the more common estrogen receptor (ER) positive subtypes. Consequently, most of the reported loci have been more strongly associated with ER-positive tumors. For example, out of the 61 new variants reported by Michailidou et al. [4], 19 showed a specific association with ER-positive breast cancer, and only two were associated with ER-negative disease. There have been growing efforts to identify variants associated with other breast cancer subtypes. Using data from over 21,000 ER-negative cases, Milne et al. [27] reported on 125 variants specifically associated with ER-negative disease. Combined, these variants were estimated to account for $16 \%$ of the familial risk for this disease subtype [27]. A strong association between ER-negative variants and breast cancer risk in BRCA1 mutation carriers was also reported [27]. Similarly to other GWAS, ER-negative variants have been mostly identified in European populations.

\section{Polygenic risk scores}

Individually, each breast cancer SNP has a minimal effect on breast cancer risk. However, their combined effect, in the form of polygenic risk scores (PRS), has been shown to provide a degree of risk discrimination that can be used to stratify individuals into different categories of disease risk. Studies examining the effect of PRS on breast cancer risk have consistently reported a higher PRS among women diagnosed with the disease when compared to population controls (Table 1) [7, 30-32, 39-42]. Overall, studies of European populations have 


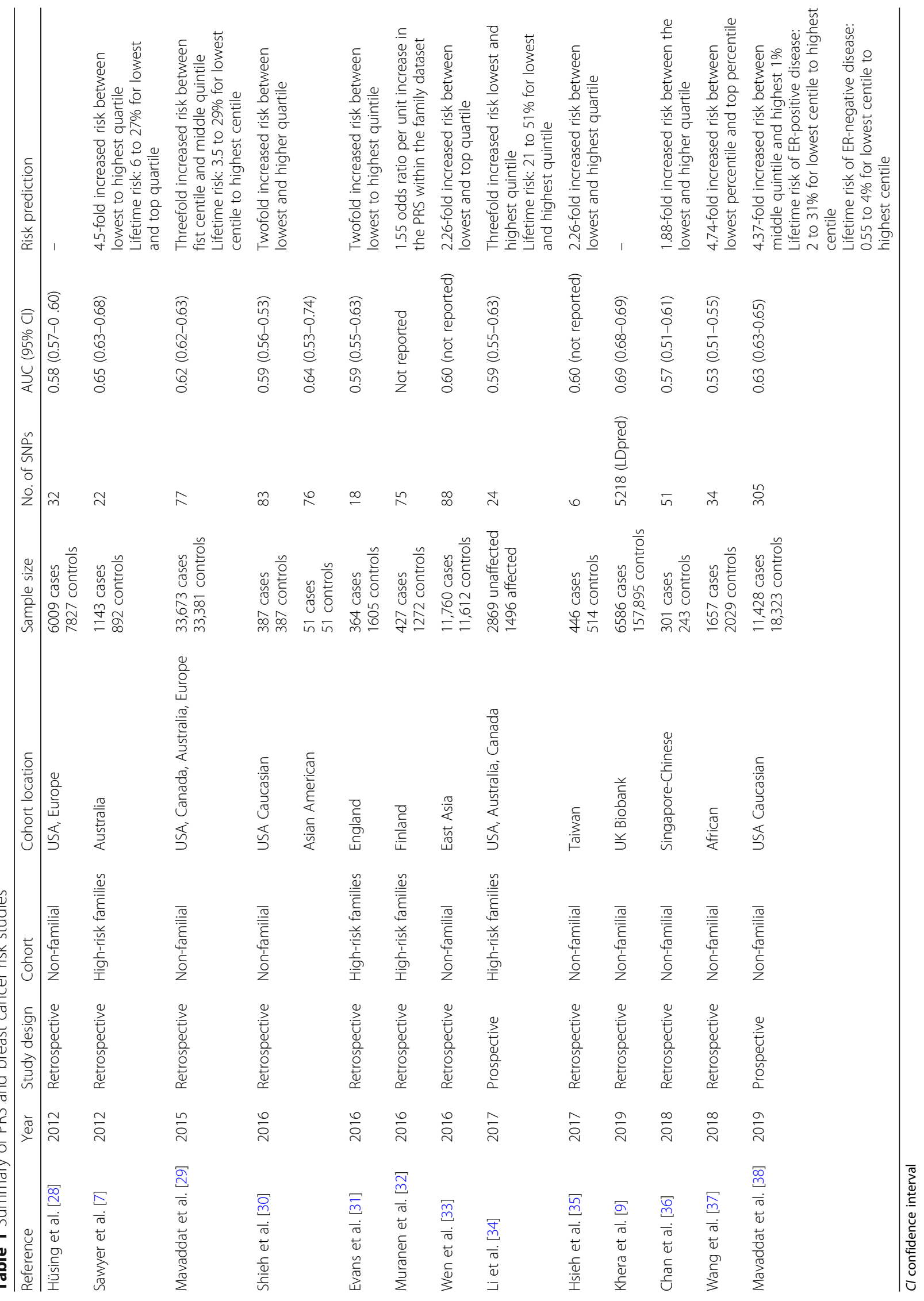


reported at least a twofold difference in breast cancer risk between the lowest and highest quartile of PRS distribution. Similar findings have also been reported across other populations including women of African American and Asian ancestry (Table 1) [26, 30, 33, 35, 36, 43]. However, not all studies have weighted the breast cancer variants for their specific ethic group, and therefore findings may not be an accurate representation of breast cancer risk for their population [35]. An increased rate of contralateral breast cancer has also been reported for women with a PRS in the highest quartile of risk distribution $[7,37,44]$.

\section{Polygenic risk and breast cancer classification}

Polygenic risk scores have been shown to differentiate risk for ER-positive and ER-negative disease. However, as noted, PRS is more effective at stratifying the risk of ER-positive disease [26, 28, 29, 32, 33, 38, 41, 45-52]. Recently, Mavaddat et al. [38] developed a PRS that was optimized for prediction of breast cancer-specific subtype. This was achieved by weighting a subset of variants according to subtype-specific effect sizes, with the remaining variants weighted for overall breast cancer risk. Improvements in risk prediction for breast cancerspecific subtypes were reported. However, prediction of ER-positive disease was still superior to ER-negative disease; odds ratio (OR) per 1 standard deviation (SD) of the PRS was 1.68 and 1.45 , respectively [38].

The possibility that PRS may provide information on prognostic factors has also been examined. In one study, a high PRS was associated with an increased risk of being diagnosed with breast cancer during routine screening rather than between screening (interval breast cancer) [48]. When compared to breast cancer diagnosed during routine screening, interval cancers are associated with poorer prognosis and more aggressive tumors [48]. Similarly, two studies reported that a high PRS was associated with more favorable tumor characteristics including lowergrade ER-positive breast cancer, smaller size tumor, and less likely to be diagnosed with distant metastasis $[45,53]$. However, these findings have not always been consistently replicated in other studies [54].

\section{Accuracy of polygenic risk models}

Several studies have assessed the discriminatory accuracy and calibration of breast cancer PRS. The discriminatory accuracy of PRS has been most commonly assessed by calculating the area under the receiver operating characteristic curve (AUC) (Table 1). The AUC is the overall probability that the predicted risk is higher for cases than for controls. Values range from 0.5 (risk for cases is higher $50 \%$ of the time, indicating the model does not discriminate cases from controls) to 1.0 (risk higher for cases $100 \%$ of the time, thus perfect discrimination) [55]. The reported AUC for breast cancer PRS has been modest, ranging from 0.58 to 0.65 for European populations and 0.53 to 0.64 for non-European populations (Table 1). Accuracy of PRS has also been reported based on breast cancer subtype, with studies reporting higher AUC for ER-positive cancer when compared to ER-negative disease $[38,46,56]$.

Calibration (i.e., an assessment of how well the model's predicted probability agrees with observed risk) was assessed in two studies, with both reporting excellent calibration for a PRS based on just 18 variants for a cohort of European ancestry [31, 41]. In another study, PRS calculated based on 75 variants was shown to be a well calibrated model for women of African American and Hispanic ancestry [39]. Finally, a study examining the effect of including PRS for risk assessment reported risk re-classification for $53 \%$ of their cohort compared to using the Manchester Scoring System alone [31], with $25 \%$ of women moving into a higher-risk category and $27 \%$ into a lower-risk category.

Determining the appropriate number of variants to include in the PRS calculation has remained a significant challenge. However, it is evident that increasing the number of variants has only had a minimal impact on the accuracy of PRS. For example, Mavaddat et al. [29] reported an AUC of 0.62 for a PRS based on 77 variants; in contrast, a PRS based on 313 variants had an AUC of 0.63 [38]. Despite the limited changes in AUC, there is evidence of improvements in the ability of the model to distribute risk in the population. Specifically, OR per 1 SD of the PRS was 1.46 for the 77 PRS [29], compared to a OR of 1.61 for the 313 PRS [38]. The modest improvement in AUC is to some extent expected with the progressive increase in the size and power of GWAS. This contributes to at least two moderating effects. Firstly, as the power of GWAS improves, variants are detected that confer lower breast cancer risk than in earlier smaller studies [57]. As a result, the additional variants that are incorporated in the PRS, add less to the overall prediction of breast cancer risk compared to the initially discovered variants in a "diminishing returns" effect. Secondly, the replication of individual SNPs or risk loci in larger GWAS allows for improved accuracy in the estimate of associated OR and can overcome the effect known as the "winners curse," whereby the first report of a SNP association tends to provide an inflated estimate of the associated risk. As a result, early estimates of the discrimination of the PRS will have a tendency for overestimation, an effect that will be corrected over time as the OR for each SNP tends to be revised downward. These problems are addressed in more recent studies that have used new statistical methods of calculation polygenic risk with the goal of improving risk prediction, 
including creating a meta-PRS [42] and using LDpred [9]. Nevertheless, further research is still needed to determine the best-performing PRS.

\section{Polygenic risk in combination with risk prediction models}

Several studies have examined the effect of PRS on existing risk prediction models, including the Gail Model [39, 46, 56, 58-62], Breast and Ovarian Analysis of Disease Incidence and Carrier Estimation $\mathrm{Al}$ gorithm (BOADICEA) [46, 63, 64], Tyrer-Cuzick (TC) [39, 41, 46, 49, 52], BRCAPRO [46], and Breast Cancer Surveillance Consortium (BCSC) $[30,40,65]$ (Table 2). These statistical models utilize a range of well-known breast cancer risk factors such as personal and family history, breast pathology, and lifestyle factors to estimate the risk of breast cancer.

Currently, all studies of European populations have reported improved accuracy when PRS is added to the model (Table 2). In a study of four different risk prediction models, Dite et al. [46] reported that across the different approaches, approximately $10 \%$ of women with a personal history of breast cancer moved into a higher breast cancer risk category and less than $2 \%$ into a lower-risk category (Table 2). Similarly, Li et al. [34] found that adding PRS to BOADICEA resulted in 16\% of their cohort moving above the risk threshold for breast screening through magnetic resonance imaging (MRI). Currently, the highest AUC has been reported in a model that combined BCSC, circulating estradiol levels, and PRS (AUC $=0.72$ ) [65]. The BCSC model uses information on age, ethnicity, first-degree family history of breast cancer, and breast density to assess breast cancer risk [65]. Such findings highlight the potential for improving current breast cancer risk discrimination through more comprehensive prediction models that include genomic information, although even the best models described to date leave room for continued improvement. As in other areas, there is a lack of studies examining these approaches in women of non-European ancestry; three relatively small studies have examined the combination of the Gail model and PRS in African American, Hispanic and Asian women respectively [30, $39,62]$ providing some evidence of improved risk prediction in these groups.

A limitation of many of these studies is that they assume independence between the PRS and previously described risk factors. Ignoring the correlation between components in the model, most obviously between genetically determined risk and family history, is likely to result in an overestimation of risk among women with a family history of the disease. A recent study by Lee et al. [66] has addressed this issue in the BOADICEA model where the effect of the residual family history is attenuated by the PRS. Future studies should aim to incorporate similar features into the other risk prediction models to address to the redundancy between family history and PRS.

\section{Applications of polygenic breast cancer risk}

Despite earlier concerns, the latest research has demonstrated that PRS is a strong predictor of breast cancer risk, with most studies of women from European ancestry suggesting a greater than twofold difference in risk between the lowest and highest PRS quartiles (Table 1). Consideration now turns to the clinical utility of PRS and challenges with clinical implementation of testing. There are several applications to polygenic breast cancer risk that include (i) to provide addition risk information to families at high-risk of breast cancer with uninformative genetic testing results, (ii) as a moderator of monogenic risk, and (iii) to guide population breast screening programs. Each application of polygenic testing has its own benefits and limitations which warrant further exploration.

\section{Familial cancer clinic}

Currently, most women at high-risk of breast cancer receive uninformative results from genetic testing of highand moderate-risk genes. Uptake of breast cancer risk management strategies among this group of women is reported to be low $[67,68]$. Thus, there is a need to develop new method of risk stratification to inform risk management decisions for women with uninformative results from monogenic testing. Studies assessing the application of polygenic factors for familial cancer have reported that PRS was predictive of breast cancer risk among women from high-risk families with uninformative $B R C A 1 / 2$ result $[7,31,32,34,46,56,64]$. Higher PRS has also been reported among women from breast cancer families when compared to those without a family history of the disease [29,31,32], suggesting that breast cancer variants may cluster in affected families. Despite some evidence of variant clustering, it is not possible to predict an individual's PRS based on their relative's result [69]. Additionally, it is likely that polygenic testing will be implemented as part of a risk prediction model such as Tyrer-Cuzick, and therefore, breast cancer risk should be evaluated individually regardless of their relatives' risk level.

Polygenic testing is likely to be implemented in familial cancer clinics, alongside an assessment of family history, for women at increased risk of breast cancer with uninformative genetic testing results. Women with uninformative result and an increased PRS could be counseled about the higher risk of early-onset breast cancer, increased risk of contralateral breast cancer, no change in ovarian cancer risk, and reduced chance of a pathogenic variant in highpenetrance genes [7]. This information can be used to 


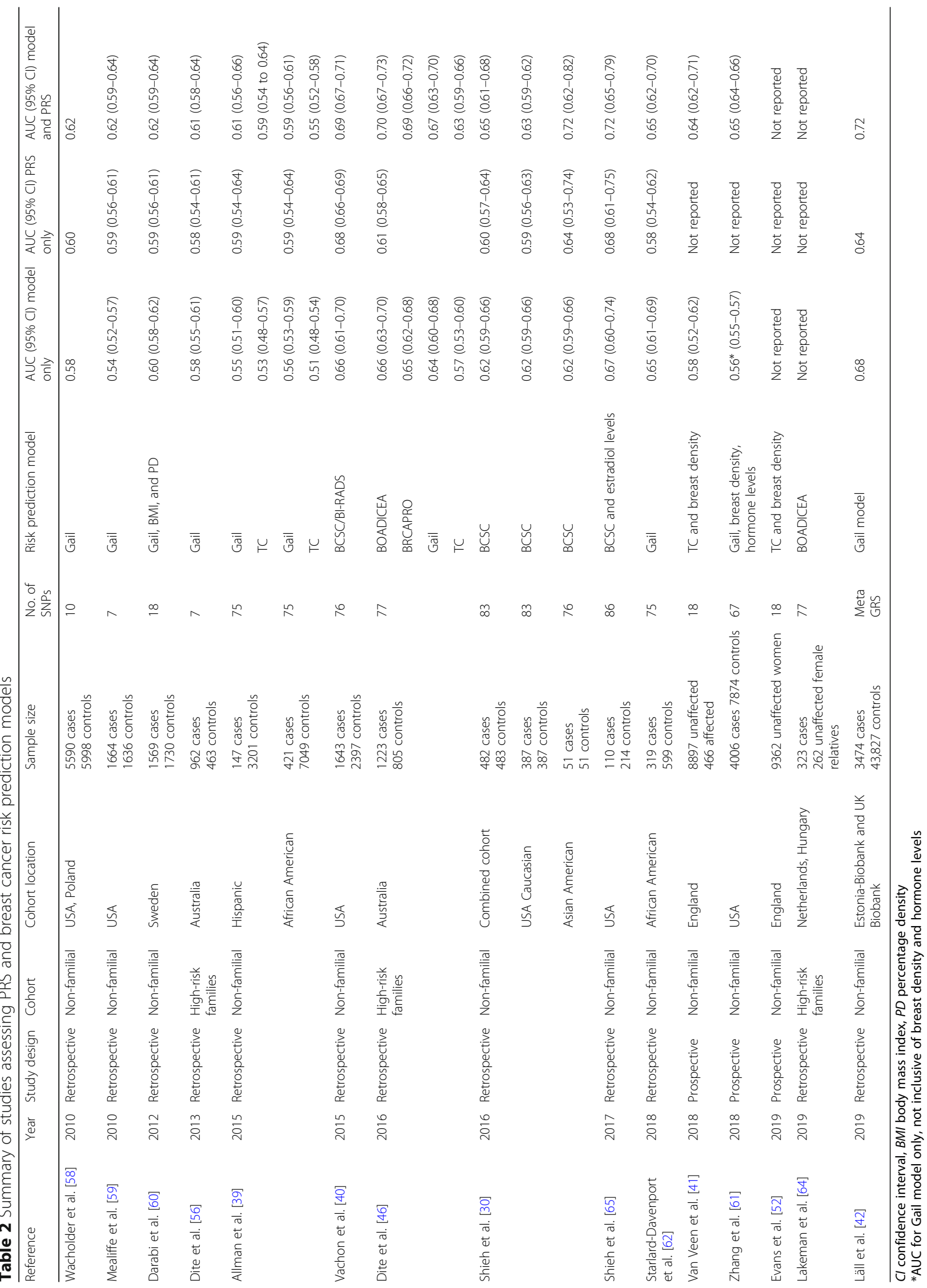


guide risk management decisions such as increased surveillance and chemoprevention. Currently, PRS is a better predictor of ER-positive breast cancer. Given that current risk-reducing medications, such as tamoxifen, function by interfering with the estrogen pathway, it is possible that this approach will be a particularly effective for women with a high PRS based on current variants [29]. Among women with a low PRS, the residual familial risk will be estimated through the integration of PRS into risk prediction models [66]. This group of women could also be informed about the ongoing risk for ER-negative disease.

Currently, there is no framework to support the implementation of breast cancer polygenic testing across clinical genetic services. Historically, genetic services have focused on testing of monogenic risk genes (e.g., $B R C A 1 / 2$ ) and its familial implications. However, a shift towards a personalized model of care will be required as polygenic testing continues to be implemented into clinical practice. While genetic counseling for common complex disorders are available [70, 71], a new model of genetic counseling that accounts for both monogenic and polygenic risk, as well as additional risk factors such as family history and lifestyle factors, is yet to be developed. Additional training of genetic health professional will also be required to ensure clinicians are able to effectively communicate this information. Furthermore, there are few studies assessing communication and response to receiving PRS [72, 73]. Research is needed to assess how women understand this complex information and the psychological and behavioral impact of receiving polygenic risk. These challenges will be magnified as polygenic testing moves into mainstream medicine. Despite the challenges, testing for breast cancer PRS is now clinically available [13, 14], with testing being target to unaffected women at increased risk of familial breast cancer with uninformative results from monogenic testing.

\section{Polygenic risk and single gene modification}

Polygenic risk scores have been shown to modify risk associated with high- and moderate-risk breast cancer risk genes [6, 74]. Using a cohort of women from the Consortium of Investigators of Modifiers of BRCA1/2 (CIMBA), Kuchenbaecker et al. [6] reported large differences in the absolute risk of developing breast cancer when PRS was incorporated in to the risk prediction model. Specifically, BRCA1 carries with a PRS in the 10th percentile of risk distribution had a $56 \%$ chance of developing breast cancer by age 80 years. In comparison, those with a PRS in the 90th percentile had a $75 \%$ breast cancer risk by age 80 years. Evidence of subtype-specific PRS was also reported, with a PRS weighted for ERnegative risk displaying the strongest association with breast cancer risk in BRCA1 carriers [6]. The AUC for these models were modest, with ER-negative PRS for BRCA1 carriers having the highest discrimination $($ AUC $=0.58)$. In another study, a PRS based on 77 variants was a significant risk factors for CHEK2*1100delC carriers [74]. Early research on the impact of PRS on monogenic risk is promising [6, 74]. However, this literature is still limited to a few studies and therefore additional research is needed.

Additional research is also needed to determine the clinical outcomes associated with implementing PRS in familial cancer clinics and guidelines for risk management strategies will need to be developed. For example, risk-reducing strategies may be recommended to CHEK2*1100delC carriers with increased risk PRS, while increased surveillance may be an appropriate strategy for those with the same pathogenic variant and reduced risk PRS. Nevertheless, if early data is replicated, incorporating PRS to high- and moderate-risk gene testing would allow for personalization of risk prediction and ultimately facilitate risk management decisions for women with pathogenic variants in these genes.

\section{Polygenic risk and population breast screening}

Despite the strong evidence for the high variability of breast cancer risk within populations, most population screening programs only utilize age as a risk factor in determining recommendations for mammographic screening. However, there is growing evidence to support the inclusion of polygenic information to population screening programs, with studies reporting an age-specific effect for PRS [9, 29, 52, 63]. For example, Mavaddat et al. [29] reported women in the 99th percentile of PRS distribution reached the threshold for population screening in their early 30 s, while women below the 20th percentile remained under the risk threshold for population screening up until age 70 years [29]. In another study, Khera et al. [9] estimated that $20 \%$ of the population would have a greater than twofold risk of developing breast cancer based on PRS alone. More recently, it was reported that greater levels of breast cancer risk stratification for the general population could be achieved by incorporating PRS, mammographic density, and other risk factors into the BOADICEA risk model [63]. The authors estimated that $13 \%$ of women in the population would be classified as moderate or high risk of developing breast cancer. Together, these data indicate that polygenic information has the potential to provide personalized risk management for a meaningful proportion of the population, including earlier and more frequent screening for women at increased risk and reduced mammographic screening for women with a lower PRS. Further research is now needed to evaluate the clinical utility of this personalized approach to population screening. For example, studies 
are needed to assess the extent in which PRS improves clinical outcomes, including reducing morbidity and mortality for at-risk women, and reducing overdiagnosis [75]. Studies are also needed to assess individual screening pathways including ages for commencing screening and modalities for calculating the absolute benefits of preventative strategies (e.g., chemoprevention) to allow women to make more informed choices about how to manage their level of risk.

Implementation of PRS into population screening programs also requires consideration of the social, ethical, and psychological outcomes. For example, consideration needs to be given to the acceptability of risk-based surveillance (particularly for those with a reduced risk), training of non-genetics health professionals, how best to communicate this information, and cost-benefit analyses [29, 75, 76]. Several largescale studies are now underway to assess the impact of implementing PRS to breast screening program including the PROCAS [52, 77], CORDIS [78], and WISDOM trial [79]. These studies will provide a platform for the rigorous evaluation of risk-based screening, as well as a framework for the implementation of PRS into population screening programs. Nevertheless, PRS testing has the potential to change population screening programs and allow access to personalized genetic testing to a wider group of women, including testing of women with breast cancer unselected for family history, and women in the general population to tailor their mammographic screening schedule.

\section{Conclusion}

Significant progress has been made in understating the genetic architecture of breast cancer and the role of polygenic information in familial breast cancer and for population screening programs. The studies presented in this review demonstrate that polygenic information has the power to effectively stratify breast cancer risk. These findings need to be considered in light of current limitations, including limited research on women from non-European ancestry and emphasis of ER-positive breast cancer. Equally, further research is needed to determine the best-performing PRS, with varying number of SNPs and statistical methods being currently used to calculate polygenic risk $[9,42]$. Despite the current limitations, testing for polygenic risk is already being implemented in specialist familial cancer clinics to provide additional information for at-risk women with uninformative genetic test results, while research is ongoing to assess the clinical utility of PRS for population screening programs.

\section{Supplementary information}

Supplementary information accompanies this paper at https://doi.org/10. 1186/s13058-020-01260-3.

Additional file 1. Breast Cancer GWAS catalog summary dated 20.10.19. This file contains a summary of all the breast cancer GWAS registered in the GWAS Catalog as of the 20th October 2019.

\section{Abbreviations \\ AUC: Area under the curve; BCAC: Cancer Association Consortium; BCSC: Breast Cancer Surveillance Consortium; BOADICEA: Breast and Ovarian Analysis of Disease Incidence and Carrier Estimation Algorithm; COGS: The Collaborative Oncological Gene-Environment Study; ER: Estrogen receptor; PRS: Polygenic risk scores; TC: Tyrer-Cuzick}

\section{Acknowledgements}

Not applicable.

\section{Authors' contributions}

TY and BM had the original idea for this article and collected the relevant data. TY searched the literature, extracted and analyzed the data, and drafted the manuscript. All authors contributed to the interpretation of the data. The author(s) read and approved the final manuscript.

\section{Funding}

TY is supported by a National Health and Medical Research Council (NHMRC) and National Breast Cancer Foundation postgraduate scholarship (ID:

1133049), and a Translational Cancer Research Institute PhD Top-up Scholarship. BM is supported by an NHMRC Senior Research Fellowship Level B (ID

1078523). The funding bodies did not have a role in the design, analysis, and interpretation of this review.

\section{Availability of data and materials}

Data sharing is not applicable to this article as no datasets were generated or analyzed during the current study.

Ethics approval and consent to participate

This article does not contain any studies with human participants or animals performed by any of the authors.

Consent for publication

Not applicable.

\section{Competing interests}

Authors TY, MAY, and PJ declare that they have no competing interests. Author BM Meiser has a remunerated consultant role with the company Astrazeneca with respect to an unrelated project.

\section{Author details}

${ }^{1}$ Psychosocial Research Group, Prince of Wales Clinical School, Faculty of Medicine, UNSW Sydney, Sydney, NSW, Australia. ${ }^{2}$ The University of Queensland Diamantina Institute, Dermatology Research Centre, University of Queensland, Brisbane, QLD 4102, Australia. ${ }^{3}$ Parkville Integrated Familial Cancer Centre, Peter MacCallum Cancer Centre, Melbourne, VIC 3000, Australia. ${ }^{4}$ Kinghorn Centre for Clinical Genomics, Garvan Institute of Medical Research, Sydney, NSW 2010, Australia.

Received: 28 June 2019 Accepted: 7 February 2020

Published online: 17 February 2020

\section{References}

1. Collaborative Group on Hormonal Factors in Breast Cancer. Familial breast cancer: collaborative reanalysis of individual data from 52 epidemiological studies including 58,209 women with breast cancer and 101,986 women without the disease. Lancet. 2001;358(9291):1389-99.

2. Lichtenstein $P$, et al. Environmental and heritable factors in the causation of cancer--analyses of cohorts of twins from Sweden, Denmark, and Finland. N Engl J Med. 2000;343(2):78-85.

3. Bahcall O. Common variation and heritability estimates for breast, ovarian and prostate cancers. Nat Genet. 2019. https://doi.org/10.1038/ngicogs.1. 
4. Michailidou $\mathrm{K}$, et al. Association analysis identifies 65 new breast cancer risk loci. Nature. 2017;551(7678):92-4.

5. Thomas DM, James PA, Ballinger ML. Clinical implications of genomics for cancer risk genetics. Lancet Oncol. 2015;16:e303-8.

6. Kuchenbaecker KB, et al. Evaluation of polygenic risk scores for breast and ovarian cancer risk prediction in BRCA1 and BRCA2 mutation carriers. J Natl Cancer Inst. 2017;109(7). https://doi.org/10.1093/jnci/djw302.

7. Sawyer S, et al. A role for common genomic variants in the assessment of familial breast cancer. J Clin Oncol. 2012;30(35):4330-6.

8. Antoniou A, et al. A response to "personalised medicine and population health: breast and ovarian cancer". Hum Genet. 2019;138(3):287-9.

9. Khera AV, et al. Genome-wide polygenic scores for common diseases identify individuals with risk equivalent to monogenic mutations. Nat Genet. 2018;50(9):1219-24.

10. Narod SA. Personalised medicine and population health: breast and ovarian cancer. Hum Genet. 2018;137(10):769-78.

11. Janssens A, Joyner MJ. Polygenic risk scores that predict common diseases using millions of single nucleotide polymorphisms: is more, better? Clin Chem. 2019;65(5):609-11.

12. Chowdhury $\mathrm{S}$, et al. Incorporating genomics into breast and prostate cancer screening: assessing the implications. Genet Med. 2013;15(6):423-32.

13. Black $M$, et al. Validation of a polygenic $r$ isk score for breast cancer in unaffected Caucasian women referred for genetic testing. J Clin Oncol. 2018;36(15_suppl):1508.

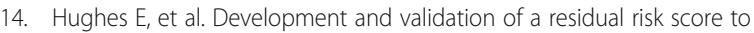
predict breast cancer risk in unaffected women negative for mutations on a multi-gene hereditary cancer panel. J Clin Oncol. 2017;35(15_suppl):1579.

15. GWAS Catalog 2018 09.01.2018]; Available from: https://www.ebi.ac.uk/gwas/.

16. Amos Cl, et al. The OncoArray consortium: a network for understanding the genetic architecture of common cancers. Cancer Epidemiol Biomark Prev. 2017;26(1):126-35

17. Breast Cancer Association Consortium. Commonly studied single-nucleotide polymorphisms and breast cancer: results from the Breast Cancer Association Consortium. J Natl Cancer Inst. 2006;98(19):1382-96. https:// pubmed.ncbi.nlm.nih.gov/17018785-commonly-studied-single-nucleotidepolymorphisms-and-breast-cancer-results-from-the-breast-cancerassociation-consortium/.

18. Bahcall, O.G. COGS project and design of the iCOGS array. 2013; Available from: https://www.nature.com/icogs/primer/cogs-project-and-design-of-the-icogs-array/.

19. Michailidou $K$, et al. Large-scale genotyping identifies 41 new loci associated with breast cancer risk. Nat Genet. 2013;45(4):353-61. 361e1-2

20. Michailidou K, et al. Genome-wide association analysis of more than 120,000 individuals identifies 15 new susceptibility loci for breast cancer. Nat Genet. 2015:47:373-80

21. Shieh $Y$, et al. A polygenic risk score for breast cancer in U.S. Latinas and LatinAmerican women. J Natl Cancer Inst. 2019. https://doi.org/10.1093/jnci/djz174.

22. Huo D, et al. Genome-wide association studies in women of African ancestry identified 3q26.21 as a novel susceptibility locus for oestrogen receptor negative breast cancer. Hum Mol Genet. 2016;25(21):4835-46.

23. Fejerman $L$, et al. Genome-wide association study of breast cancer in Latinas identifies novel protective variants on 6q25. Nat Commun. 2014;5:5260.

24. Lee JY, et al. BRCA1/2-negative, high-risk breast cancers (BRCAX) for Asian women: genetic susceptibility loci and their potential impacts. Sci Rep. 2018;8(1):15263.

25. Hoffman J, et al. Identification of novel common breast cancer risk variants at the 6 q25 locus among Latinas. Breast Cancer Res. 2019;21(1):3.

26. Wang $\mathrm{S}$, et al. Genetic variants demonstrating flip-flop phenomenon and breast cancer risk prediction among women of African ancestry. Breast Cancer Res Treat. 2018:168(3):703-12.

27. Milne RL, et al. Identification of ten variants associated with risk of estrogenreceptor-negative breast cancer. Nat Genet. 2017:49(12):1767-78.

28. Husing $A$, et al. Prediction of breast cancer risk by genetic risk factors, overall and by hormone receptor status. J Med Genet. 2012;49(9):601-8.

29. Mavaddat $\mathrm{N}$, et al. Prediction of breast cancer risk based on profiling with common genetic variants. J Natl Cancer Inst. 2015;107(5). https://doi.org/10. 1093/jnci/djv036.

30. Shieh $Y$, et al. Breast cancer risk prediction using a clinical risk model and polygenic risk score. Breast Cancer Res Treat. 2016;159(3):513-25.

31. Evans DG, et al. The impact of a panel of 18 SNPs on breast cancer risk in women attending a UK familial screening clinic: a case-control study. J Med Genet. 2016;54(2):111-13.
32. Muranen TA, et al. Polygenic risk score is associated with increased disease risk in 52 Finnish breast cancer families. Breast Cancer Res Treat. 2016: 158(3):463-69.

33. Wen $\mathrm{W}$, et al. Prediction of breast cancer risk based on common genetic variants in women of east Asian ancestry. Breast Cancer Res. 2016;18(1):124.

34. Li H, et al. Breast cancer risk prediction using a polygenic risk score in the familial setting: a prospective study from the Breast Cancer Family Registry and kConFab. Genet Med. 2017;19(1):30-5.

35. Hsieh YC, et al. A polygenic risk score for breast cancer risk in a Taiwanese population. Breast Cancer Res Treat. 2017;163(1):131-8.

36. Chan $\mathrm{CHT}$, et al. Evaluation of three polygenic risk score models for the prediction of breast cancer risk in Singapore Chinese. Oncotarget. 2018; 9(16):12796-804.

37. Wang Z, et al. Polygenic determinants for subsequent breast cancer risk in survivors of childhood cancer: The St Jude Lifetime Cohort Study (SJLIFE). Clin Cancer Res. 2018;24(24):6230-35.

38. Mavaddat $\mathrm{N}$, et al. Polygenic risk scores for prediction of breast cancer and breast cancer subtypes. Am J Hum Genet. 2019;104(1):21-34.

39. Allman $\mathrm{R}$, et al. SNPs and breast cancer risk prediction for African American and Hispanic women. Breast Cancer Res Treat. 2015;154(3):583-9.

40. Vachon CM, et al. The contributions of breast density and common genetic variation to breast cancer risk. J Natl Cancer Inst. 2015;107(5). https://doi. org/10.1093/jnci/dju397.

41. van Veen EM, et al. Use of single-nucleotide polymorphisms and mammographic density plus classic risk factors for breast cancer risk prediction. JAMA Oncol. 2018;4(4):476-82.

42. Lall $\mathrm{K}$, et al. Polygenic prediction of breast cancer: comparison of genetic predictors and implications for risk stratification. BMC Cancer. 2019;19(1):557.

43. Lee $C P$, et al. Breast cancer risk assessment using genetic variants and risk factors in a Singapore Chinese population. Breast Cancer Res. 2014;16(3):R64.

44. Robson ME, et al. Association of common genetic variants with contralateral breast cancer risk in the WECARE Study. J Natl Cancer Inst. 2017;109(10). https://doi.org/10.1093/jnci/djx051.

45. Holm J, et al. Associations of breast cancer risk prediction tools with tumor characteristics and metastasis. J Clin Oncol. 2016;34(3):251-8.

46. Dite GS, et al. Breast cancer risk prediction using clinical models and 77 independent risk-associated SNPs for women aged under 50 years: Australian breast cancer family registry. Cancer Epidemiol Biomark Prev. 2016;25(2):359-65.

47. Vachon $\mathrm{CM}$, et al. A polygenic risk score for breast cancer in women receiving tamoxifen or raloxifene on NSABP P-1 and P-2. Breast Cancer Res Treat. 2015;149(2):517-23.

48. Li J, et al. Breast cancer genetic risk profile is differentially associated with interval and screen-detected breast cancers. Ann Oncol. 2015;26(3):517-22.

49. Cuzick J, et al. Impact of a panel of 88 single nucleotide polymorphisms on the risk of breast cancer in high-risk women: results from two randomized tamoxifen prevention trials. J Clin Oncol. 2017;35(7):743-50.

50. Rudolph A, et al. Joint associations of a polygenic risk score and environmental risk factors for breast cancer in the breast cancer association consortium. Int J Epidemiol. 2018;47(2):526-36.

51. Li N, et al. Evaluating the breast cancer predisposition role of rare variants in genes associated with low-penetrance breast cancer risk SNPs. Breast Cancer Res. 2018;20(1):3.

52. Evans DGR, et al. Breast cancer pathology and stage are better predicted by risk stratification models that include mammographic density and common genetic variants. Breast Cancer Res Treat. 2019;176(1):141-8.

53. Li J, et al. Differential burden of rare and common variants on tumor characteristics, survival, and mode of detection in breast cancer. Cancer Res. 2018;78(21):6329-38.

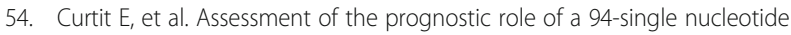
polymorphisms risk score in early breast cancer in the SIGNAL/PHARE prospective cohort: no correlation with clinico-pathological characteristics and outcomes. Breast Cancer Res. 2017;19(1):98.

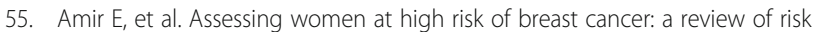
assessment models. J Natl Cancer Inst. 2010;102(10):680-91.

56. Dite GS, et al. Using SNP genotypes to improve the discrimination of a simple breast cancer risk prediction model. Breast Cancer Res Treat. 2013;139(3):887-96.

57. Wang WYS, et al. Genome-wide association studies: theoretical and practical concerns. Nat Rev Genet. 2005;6(2):109-18.

58. Wacholder $\mathrm{S}$, et al. Performance of common genetic variants in breastcancer risk models. N Engl J Med. 2010;362(11):986-93. 
59. Mealiffe ME, et al. Assessment of clinical validity of a breast cancer risk model combining genetic and clinical information. J Natl Cancer Inst. 2010; 102(21):1618-27.

60. Darabi $\mathrm{H}$, et al. Breast cancer risk prediction and individualised screening based on common genetic variation and breast density measurement. Breast Cancer Res. 2012;14(1):1-11.

61. Zhang $X$, et al. Addition of a polygenic risk score, mammographic density, and endogenous hormones to existing breast cancer risk prediction models: a nested case-control study. PLoS Med. 2018;15(9):e1002644.

62. Starlard-Davenport A, et al. Validation of a genetic risk score for Arkansas women of color. PLoS One. 2018;13(10):e0204834.

63. Lee $A$, et al. BOADICEA: a comprehensive breast cancer risk prediction model incorporating genetic and nongenetic risk factors. Genet Med. 2019; 21(8):1708-18.

64. Lakeman IMM, et al. Addition of a 161-SNP polygenic risk score to family history-based risk prediction: impact on clinical management in nonBRCA1/2 breast cancer families. J Med Genet. 2019;56(9): 581-89.

65. Shieh $Y$, et al. Joint relative risks for estrogen receptor-positive breast cancer from a clinical model, polygenic risk score, and sex hormones. Breast Cancer Res Treat. 2017;166(2):603-12

66. Lee A, et al. BOADICEA: a comprehensive breast cancer risk prediction modelincorporating genetic and nongenetic risk factors. Genet Med. 2019; 21(8):1708-18

67. Schwartz MD, et al. Long-term outcomes of BRCA1/BRCA2 testing: risk reduction and surveillance. Cancer. 2012;118(2):510-7.

68. Morgan D, et al. Cancer prevention and screening practices among women at risk for hereditary breast and ovarian cancer after genetic counseling in the community setting. Familial Cancer. 2009;8(4):277-87.

69. Cox DG, et al. Transmission of breast cancer polygenic risk based on single nucleotide polymorphisms. Breast. 2018;41:14-8.

70. Waxler JL, et al. Genetic counseling as a tool for type 2 diabetes prevention: a genetic counseling framework for common polygenetic disorders. J Genet Couns. 2012:21(5):684-91.

71. Peay $\mathrm{H}$, Austin J. How to talk with families about: genetics and psychiatric illness. In: How to talk with families about: genetics and psychiatric illness, vol. xii. New York: W W Norton \& Co; 2011. p. 266. xii, 266

72. Young MA, et al. Making sense of SNPs: women's understanding and experiences of receiving a personalized profile of their breast cancer risks. J Genet Couns. 2018;27(3):702-8.

73. Forrest $L E$, et al. High-risk women's risk perception after receiving personalized polygenic breast cancer risk information. J Community Genet. 2019;10(2):197-206.

74. Muranen TA, et al. Genetic modifiers of CHEK2*1100delC-associated breast cancer risk. Genet Med. 2017;19(5):599-603.

75. Pashayan $\mathrm{N}$, et al. Cost-effectiveness and benefit-to-harm ratio of riskstratified screening for breast cancer: a life-table model. JAMA Oncol. 2018, 4(11):1504-10

76. Henneman $L$, et al. 'A low risk is still a risk': exploring women's attitudes towards genetic testing for breast cancer susceptibility in order to target disease prevention. Public Health Genomics. 2011;14(4-5):238-47.

77. Evans DG, et al. Programme Grants for Applied Research. In: Improvement in risk prediction, early detection and prevention of breast cancer in the NHS Breast Screening Programme and family history clinics: a dual cohort study. Southampton: NIHR Journals Library; 2016.

78. Shieh, $Y$., et al., Breast cancer screening in the precision medicine era: riskbased screening in a population-based trial. J Natl Cancer Inst. 2017;109(5). https://doi.org/10.1093/jnci/djw290.

79. Esserman $\sqcup$, et al. The WISDOM Study: breaking the deadlock in the breast cancer screening debate. NPJ Breast Cancer. 2017;3(1):34.

\section{Publisher's Note}

Springer Nature remains neutral with regard to jurisdictional claims in published maps and institutional affiliations. 